\title{
Gestión del conocimiento científico-técnico contable en la Facultad de Ciencias Contables de la UNMSM con la empresa
}

\author{
Dr. Saturnino Alfonso Ugarte Silva \\ Docente Principal de la Facultad de Ciencias Contables
}

Mg. Elsa Esther Choy Zevallos

Docente Asociada de la Facultad de Ciencias Contables

\section{RESUMEN:}

Las universidades son parques cientificos que permiten producir conocimiento; mayormente, es individual a travès del desarrollo de tesis para optar el titulo profesional, grado de maestría o doctorado; sin embargo, esta información escrita solo queda en la institución y no existe una adecuada base de datos que permita conocerla en su totalidad e intercambiar conocimientos con otras instituciones academicas y empresariales.

Por otro lado, es importante tener en cuenta la capacidad limitada del cerebro humano en adquirir, almacenar y procesar conocimiento, por tanto la creación de parques tecnológicos permitirá determinar el diseño, la construcción y proceso de todo el conocimiento existente en las universidades, específicamente en las facultades de ciencias contables, facilitando la transmisión del conocimiento y base del "conocer".

Este artículo pretente priorizar la importancia de aplicar un modelo de Gestión del Conocimiento y en particular para la Facultad de Ciencias Contables de la Universidad Nacional Mayor de San Marcos, que permita su análisis y relación con procesos tecnológicos adecuados, y de esta manera fomentar el desarrollo de más conocimiento, con valor agregado, y que el mismo se incorpore a las instituciones académicas y empresariales; además de señalar la necesidad de aplicar gestión del conocimiento como herramienta gerencial en el escenario universidad-empresa y establecer la importancia de utilizar la tecnología como herramienta de información en el vínculo universidad-empresa en los procesos esenciales de la universidad.

Palabras clave: Conocimiento, gestión de conocimiento, universidad, empresa, tecnología. 


\section{INTRODUCCIÓN}

El siglo XXI, considerado la era del conocimiento, evidencia un claro desfase de la universidad con la época, esta requiere de un cambio enmarcado dentro del campo de la mejora continua de la calidad de la enseñanza y de la calidad de la investigación, ambas constituyen la base para aspirar a la excelencia institucional.

En una sociedad basada en el conocimiento, como se ha dado en llamar en la actualidad, la universidad se convierte en un elemento clave del sistema de innovación, investigación e inclusión, tanto como proveedora de capital humano y como promotora de nuevas empresas y tecnologías.

En este sentido, el papel de la universidad en la generación de conocimiento científicotécnico es que a medida que el conocimiento adquiera una importancia creciente en la innovación, la universidad, como institución que produce conocimiento, debería desempeñar un protagonismo mayor en la innovación empresarial

Para la Universidad Nacional Mayor de San Marcos, supone la necesidad de que sus Facultades se integren en redes nacionales e internacionales y establezcan alianzas estratégicas con otros entes educativos, considerando que la universidad tiene una política de autoevaluación permanente.

El propósito del presente artículo es señalar que la Facultad de Ciencias Contables de la UNMSM tiene como tarea prioritaria promover la trasmisión de conocimiento con el vínculo universidad y empresa, presentando un modelo de gestión de conocimiento que permita establecer los conocimientos contables que se producen y proyectarlos hacia los problemas y potencialidades de desarrollo del entorno socioeconómico y regional.

\section{Análisis situacional}

- ¿En los entornos actuales, la universidad reconoce a la empresa como parte de su entorno?

- ¿Necesita la empresa la participación de la universidad para su sostenibilidad?

\section{Justificación}

Existe una tendencia creciente en el mundo a la transferencia de conocimientos y de tecnología del sistema público de investigación y desarrollo hacia el sector empresarial.

Para la Facultad de Ciencias Contables supone la necesidad de que sus investigadores se integren en redes nacionales e internacionales y establezcan alianzas estratégicas con otros centros educativos y empresariales.

\section{TEORÍAS DE LA INVESTIGACIÓN}

Fundamentos epistemológicos de dirección del conocimiento organizativo: desarrollo, edición y gestión de intangibles (Eduardo Bueno Campos)

Establece que el enfoque basado en conocimiento es un nuevo paradigma para entender la economía y las organizaciones que la componen en su estructura, comportamiento y evolución, gracias al análisis de los procesos de conocimiento, como expresión de las actividades que le ponen en acción y justifican la transformación económica o los procesos que crean, desarrollan, miden y gestionan los activos o recursos tangibles - basados en conocimiento- que protagonizan la nueva realidad socioeconómica.

Bioeconomía: simbiosis científica de complejidad, organismos y comportamientos (Eduardo Bueno Campos)

Señala cómo se construye el conocimiento científico y, lógicamente, el humano gracias 
a la convergencia y aportaciones de ciencias antes separadas o poco relacionadas, ahora conectadas e integradas en una nueva concepción sistémica de la ciencia o en una «nueva alianza científica», observable por formas de investigación de grupos interdisciplinares, en los que biólogos, químicos y físicos junto a otros científicos: matemáticos, cibernéticos y de las ciencias sociales, entre otros, hacen de la ciencia su oficio, conversan, colaboran y crean conocimiento, unos con otros, en proyectos complejos, de naturaleza tecnocientífica y pluridisciplinar.

Hacia la sociedad del conocimiento (Informe mundial de la UNESCO)

Educación, ciencia, cultura y comunicación: la amplitud del ámbito abarcado por la UNESCO es, a un tiempo, una garantía de la pertinencia de su misión y una muestra de la creciente complejidad de esta.

La tercera misión de la universidad: la transferencia del conocimiento - base cognoscitiva- (Eduardo Bueno, 2007)

Teniendo en cuenta que la primera misión de la universidad es la enseñanza y el aprendizaje, y la segunda es la investigación; corresponde a la tercera misión de la universidad el emprendimiento, la innovación y el compromiso social.

Señala que los actores participantes como agentes sociales del conocimiento son las universidades, como propias del sistema científico, y las empresas, propias del sistema tecnológico.

\section{LA GESTIÓN DEL CONOCIMIENTO EN LA EMPRESA}

A pesar del fuerte interés que existe en la gestión del conocimiento por parte de los

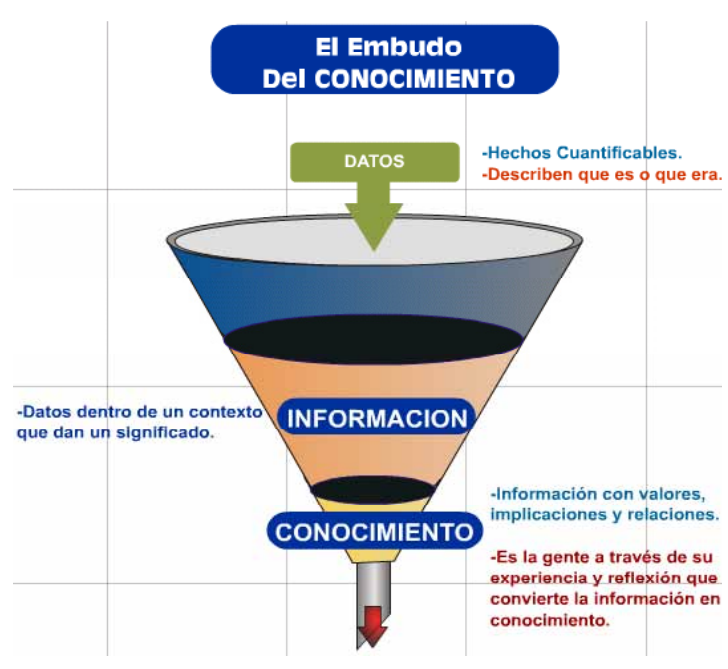

empresarios, todavía se carece de una teoría estructurada sobre la misma. Si bien se han hecho grandes avances en la última década, prestando mayor atención a los recursos intangibles, desarrollando herramientas como el benchamrking, modelos de calidad total, reingeniería, de procesos, etc., donde implícitamente se reconoce que lo realmente importante en una organización no son tanto los recursos tangibles que posee, sino la forma en que se usan y combinan. Es necesario sintetizar estos conceptos, desarrollar metodologías de análisis, sistemas de medición, etc., para que se pueda hablar propiamente de una teoría de empresa basada en el conocimiento. La gestión del conocimiento ha surgido en la empresa y en las grandes empresas de consultorio, debido a tres fuerzas económicas:

- Rápida obsolescencia de la base de competencias.

- Urgencia por valorar intangibles, en gran parte por la ola de fusiones y adquisiciones.

- Integración de soluciones de tecnologías de información.

En tal sentido, en las revistas científicas especializadas en gestión del conocimien- 
to, se suele exponer experiencias exitosas o errores a evitar en aspectos relacionados con la gestión del conocimiento, que describen puntos de vista u opiniones personales, comenzándose a trabajar también en la búsqueda de modelos integradores.

La gestión de conocimiento en la empresa está representada por la planificación, organización, coordinación y control de las actividades que lleven a la captura, creación y difusión del conocimiento en la empresa u otro tipo de organización de una manera eficiente

En tal sentido, las actividades relacionadas con la gestión del conocimiento están contenidas en la generación de nuevo conocimiento, el acceso a conocimiento valioso del exterior, la explicitación del conocimiento en base de datos, software, documentos, etc.; la transferencia de conocimiento a otras partes de la organización, la medición del valor del conocimiento disponible; el establecimiento de incentivos adecuados para que el conocimiento se cree y difunda entre los miembros de la organización; la transformación de la cultura de la organización hacia una que facilite el crecimiento del conocimiento.

Autores como Spender (1996), Grant (1996), Kogut y Zander (1992) o Teece (1998), entre otros, intentan construir una teoría de la empresa basada en el conocimiento, como una de las líneas estratégicas de investigación.

Y se ha llegado a la conclusión que si el conocimiento de un individuo no se comparte con otros miembros de la organización, el conocimiento de este es mucho menos efectivo. Por ello, una de las tareas importantes de la gestión del conocimiento es la de facilitar las interacciones entre los miembros de la organización, en primer lugar, y luego de forma externa a otras organizaciones e instituciones.
En síntesis, la gestión del conocimiento consta de una serie de subprocesos: creación de conocimiento, adopción de conocimiento, distribución de conocimiento y revisión de conocimiento.

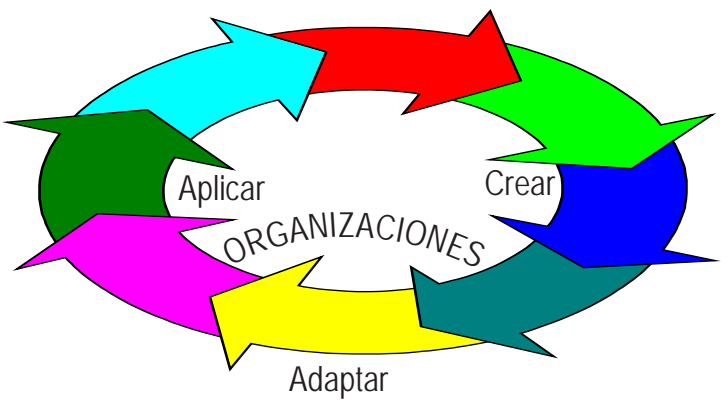

\section{EL NUEVO PAPEL DE LA UNIVERSIDAD EN LA CREACIÓN Y GESTIÓN DEL CONOCIMIENTO CIENTÍFICO TÉCNICO}

En una sociedad basada en el conocimiento, como se ha dado en llamar, la universidad se convierte en un elemento clave del sistema de innovación, investigación e inclusión.

La propuesta del papel de la universidad en la generación de conocimiento científicotécnico es que a medida que el conocimiento vaya teniendo una importancia creciente en la innovación, la universidad, como institución que produce conocimiento, debería desempeñar un protagonismo mayor en la innovación empresarial, por las siguientes razones:

- En primer lugar. Es evidente que se pretende estimular la competencia y la cooperación entre empresas, centros tecnológicos y universidades en la financiación de la investigación.

- En segundo lugar. Existe una tendencia creciente en el mundo a la transferencia de conocimientos y de tecnología, del sistema público de investigación y desarrollo hacia el sector empresarial. 
En conclusión, la gestión de conocimiento debe ser una competencia gerencial cuya finalidad sea crear las condiciones para asegurar que los planes estratégicos y políticas de aseguramiento en calidad e investigación y los procesos de la Universidad estén dirigidos y coordinados para generar los niveles deseados de conocimiento en beneficio de la sociedad y la comunidad universitaria.

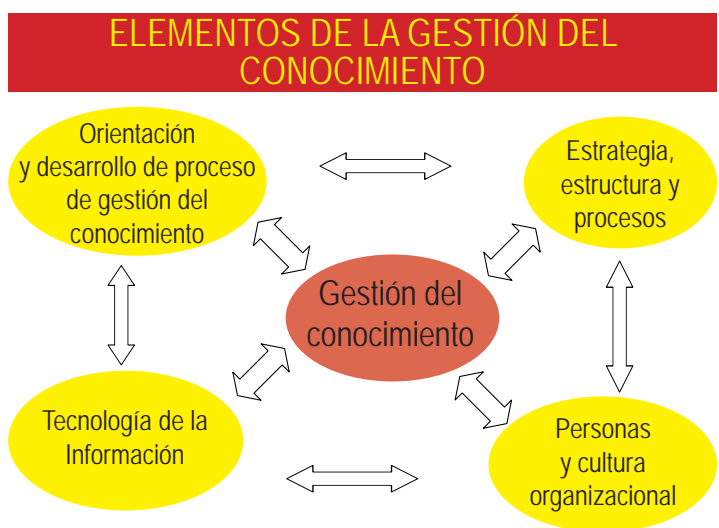

\section{LA UNIVERSIDAD PERUANA}

El siglo XXI, considerado la era del conocimiento, evidencia un claro desfase de la universidad con la época, esta requiere de un cambio enmarcado dentro del campo de la mejora continua de la calidad de la enseñanza y de la calidad de la investigación, ambos constituyen la base para aspirar a la excelencia institucional.

La mejora permanente de la calidad de la enseñanza permitirá que los alumnos estén preparados con métodos más modernos, que utilicen la tecnología de la información más adecuada y en especial que mejoren su formación académica para que egresen con la capacidad de crear nuevos conocimientos y aplicarlo en las empresas, así como resolver problemas de la sociedad.

Teniendo en cuenta que la universidad es un sistema de conocimiento, debe tener las siguientes funciones:
- Función investigadora: Crea conocimiento.

- Función educadora: Transmite y forma conocimiento.

- Función gestora: Administra conocimiento.

La gestión de conocimiento en la universidad comprende:

\section{Gestión académica administrativa}

- Automatización de procesos académicos.

- Relación académica estudiante-universidad.

\section{Portales e intranets}

- Sistemas de racionalización administrativa.

\section{Dirección estratégica}

- Plan estratégico.

- Sistema de autoevaluación y acreditación.

- Sistemas de contabilidad.

- Sistemas de apoyo para toma de decisiones.

\section{Docencia y aprendizaje}

- Sistemas de información.

- Repositorios de materiales educativos.

- Aula virtual.

- Comunidades de práctica y aprendizaje.

\section{Investigación}

- Acceso a recursos de información $\rightarrow$ biblioteca digital.

- Identificación de capacidad científica.

- Fomento de grupos y redes de investigación.

- Promoción y difusión de resultados de investigación.

La finalidad de hacer gestión del conocimiento en la universidad están referidos a:

- Crear un sistema de producción, conservación, organización y circulación de conocimiento.

- Destinado a la mejora y la efectividad de:

- La toma de decisiones.

- La resolución de procesos estratégicos de negocio. 
- En las tres dimensiones del conocimiento:

- Su producción (investigación).

- Su formación profesional.

- Su administración.

\section{Vínculo universidad empresa en el proceso de innovación}

- Permite los procesos de innovación desde la perspectiva de los entornos científicotecnológicos.

- Promueve las actividades de interfase.
- Determinante como intermediación y externalización para facilitar la transmisión de conocimientos bidireccionales entre ambas organizaciones.

\section{Facultad de Ciencias Contables de la Uni- versidad Nacional Mayor de San Marcos}

\section{El artículo $1^{\circ}$ del Estatuto de la UNMSM} señala: «La Universidad Nacional Mayor de San Marcos, Decana de América, es la institución de mayor jerarquía académica, sus-

INSUFICIENTE VÍNCULO UNIVERSIDAD EMPRESA (VUE)

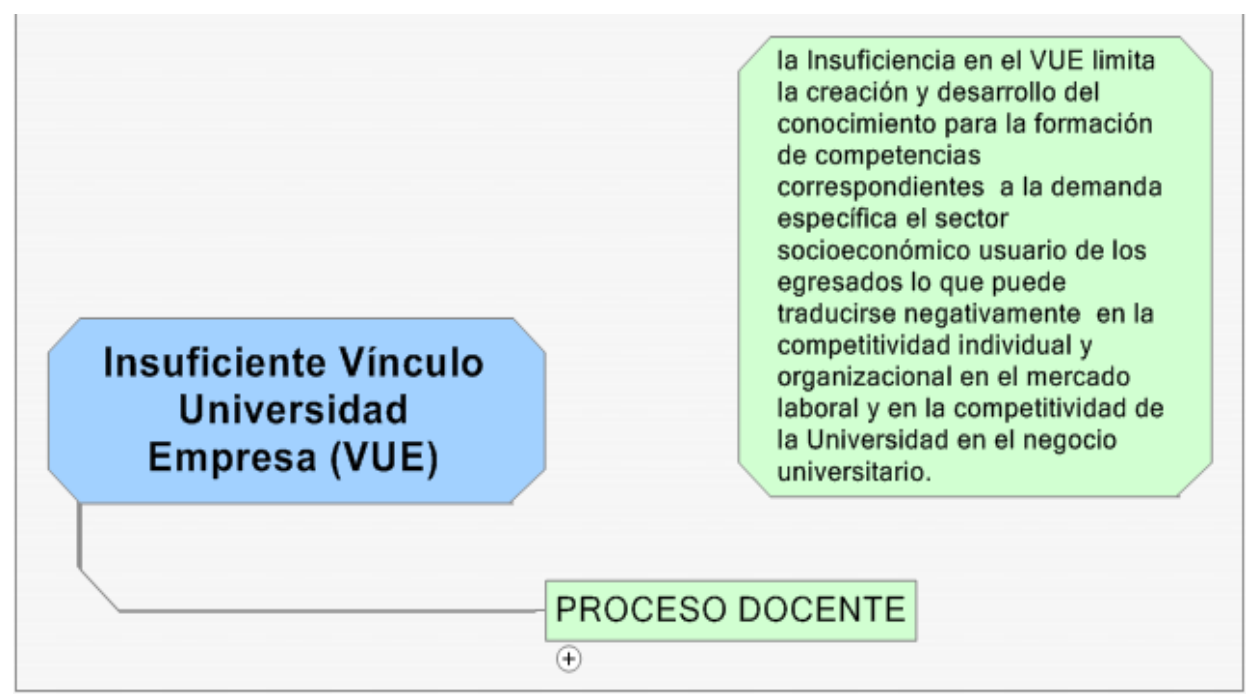

INSUFICIENTE VÍNCULO UNIVERSIDAD EMPRESA (VUE)

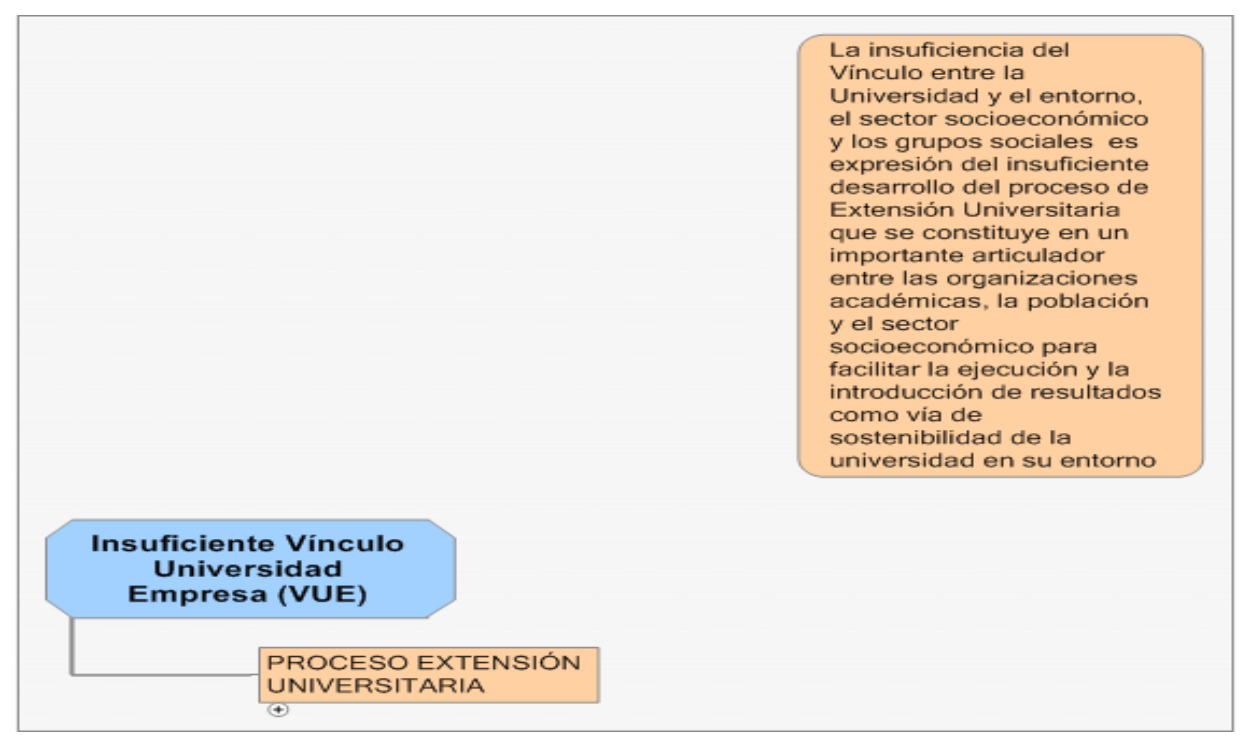


tentada en su historia y en su desarrollo, a la que la nación encarga crear y difundir conocimiento, cultura, ciencia y tecnología, con una definida orientación nacional y democrática, comprometida con la transformación de la sociedad".

\section{Modelo de gestión de conocimiento para la Facultad de Ciencias Contables de la UNMSM}

La gestión del conocimiento debe partir por la misión y visión de la universidad; en este sentido, la Facultad de Ciencias Contables de la UNMSM presenta los siguientes aspectos:

\section{Misión}

Somos una Facultad dedicada a la enseñanza, formación, especialización e investigación en las Ciencias Contables y Empresariales comprometidos con el desarrollo económico y social del país.

\section{Visión}

Ser una facultad líder y reconocida a nivel internacional en la formación, investigación e innovación de las Ciencias Contables y Empresariales.

\section{Creación del valor}

Los resultados deben ser usados para crear y equilibrar valores para los estudiantes y partes relacionadas entre la universidad, la comunidad y el Estado, para contribuir a mejorar el desempeño global de la educación.

Principales ejes de politica estratégica de la UNMSM-FCC

- Académico

- Investigación

- Extensión y alianzas estratégicas

- Gestión innovadora

- Descentralización

\section{Objetivos generales}

\section{ACADÉMICO}

Formar profesionales contables y financieros a nivel de pregrado y postgrado, acorde a las demandas de las instituciones privadas y públicas a nivel nacional e internacional, con conocimientos de última generación en concordancia con los estándares de acreditación, y contar con docentes investigadores del más alto nivel académico en el medio nacional e internacional, con compromiso ético y moral, que contribuyan a la reingeniería y gestión organizacional de la Facultad y a la formación y perfeccionamiento de profesionales integrales.

\section{EN INVESTIGACIÓN}

Lograr un óptimo desarrollo de las investigaciones en los campos de la epistemología contable, contabilidad aplicada e interpretación de la información contable para la gestión y toma de decisiones orientadas a la solución de problemas fundamentales de la sociedad, mediante alianzas y convenios internos y externos, generativos de innovaciones tecnológicas que favorezcan la competitividad empresarial en el mercado nacional e internacional.

EN EXTENSIÓN Y ALIANZAS ESTRATÉGICAS Hacer de la integración social un pilar para el desarrollo de la universidad, estableciendo canales de interacción entre universidad, Estado, empresa e instituciones sociales, hacia un desarrollo integral y sostenible.

\section{EN GESTIÓN INNOVADORA}

Alcanzar un efectivo funcionamiento académico-administrativo de la Facultad de Ciencias Contables, como resultado de la aplicación de una reingeniería en los campos de la enseñanza-aprendizaje, auditoría en los 
procesos y unificación e integración de los sistemas a través de la sensibilización de cada uno de los integrantes de la Facultad, que permita un sistema administrativo eficiente y de alta calidad que logre la acreditación de la Facultad.

\section{EN DESCENTRALIZACIÓN}

Lograr que la Facultad se descentralice a través de nuevas sedes (San Juan de Lurigancho) y extensión de sus cursos a provincias y a nivel internacional.

\section{Sistema de control}

\section{Académico}

- Acreditación de la Facultad de Ciencias Contables.

- Número de Convenios Internacionales / Número de investigadores.

- Número de Tesis de Doctorado / Número de Doctorandos.

- Número de actualizaciones realizadas al plan de estudios.

- Número de actividades sociales, culturales, artística y deportivas.

- Número de docentes capacitados / Número de docentes.

- Programa de comunicación con nuestros egresados.

- Número de programas de enseñanza a distancia implementados.

2. Investigación

- Número de programas de capacitación para investigación para docentes.

- Número de graduados a través de tesis / Número de egresados.

- Número de proyectos de investigación al año.

- Número de graduados en postgrado / Número de egresados.

\section{Extensión y alianzas estratégicas}

- Centro de producción implementado.

- Número de cursos a la sociedad implementados al ańo.

- Número de acciones con los egresados.

- Número de actividades culturales al ańo.

\section{Gestión innovadora}

- Número de programas de sensibilización.

- Número de personal capacitado / Número de personal administrativo.

- Número de reformas en la infraestructura.

\section{Descentralización}

- Número de convenios implementados al año.

- Número de mecanismos de financiamiento al año.

- Número de políticas de inclusión.

- Número de eventos científicos y tecnológicos.

Todos estos indicadores deben presentar resultados óptimos si verdaderamente queremos mejorar la gestión de conocimiento entre universidad y empresa.

\section{CONCLUSIONES}

- El conocimiento y la información producida en las acciones sociales desarrolladas por la Universidad se captura pero no se sistematiza ni se difunde, por lo que no tributa al fortalecimiento del vínculo entre la universidad y los actores sociales ni a la toma de decisiones.

- Los proyectos de investigación vinculados con la empresa parten sustancialmente de los intereses de la Universidad. Es baja la producción de resultados de investigación en el sector empresarial. 
- Insuficiente desarrollo de acciones conjuntas para el intercambio de resultados de investigación, desarrollo e innovación, entre la Universidad y la empresa.

- Escasa cultura en los docentes para el desarrollo de proyectos conjuntos aprovechando recursos financieros de ambos sectores.

- Carencia de un sistema de vigilancia tecnológica acerca de los problemas del sector empresarial susceptibles de ser objeto de investigación universitaria.

\section{RECOMENDACIONES}

- Las universidades deben difundir el conocimiento y la información que producen, hacer gestión del conocimiento con enfoque gerencial constituye una herramienta determinante para el vínculo Universidad-empresa

- La Universidad debe ir más allá de la misión de la enseñanza e investigación científica, debe basar su visión hacia la extensión de sus actividades y el desarrollo económico y social de su comunidad o entorno de referencia.

- La Universidad debe desarrollar políticas y acciones que le permita ser más competente y competitiva en su misión a nivel internacional.

- La Universidad debe promover la creación de la cultura científica y de innovación que necesita la sociedad actual.

- La Universidad debe pasar de la creación de conocimiento de forma autosuficiente o con escasa cooperación con su entorno a un nuevo modo en colaboración o abierto a través de las redes y relaciones con otros agentes del sistema de ciencia, tecnología y sociedad.

\section{REFERENCIAS}

Artiles Visbal, Leticia. Material didáctico gestión del conocimiento. Fundación Iberoamericana del Conocimiento.

Bueno Campos, Eduardo. La gestión del conocimiento científico y técnico en la comunidad de Madrid. Un proyecto en marcha. Madrid, Universidad Autónoma de Madrid.

Bueno Campos, Eduardo. Gestión del conocimiento en universidades y organismos públicos de investigación. Madrid, Ediciones de la Dirección General de Investigación - Consejería de Educación.

Bueno Campos, Eduardo. Bioeconomía: Simbiosis científica de complejidad, organismos y comportamiento.

Bueno Campos, Eduardo. Fundamentos epistemológicos de dirección del conocimiento organizativo: desarrollo, medición y gestión de intangibles

InFORME Mundial de LA UNESCO. Hacia la sociedad del conocimiento.

Miguel A., Esteban. La gestión del conocimiento en las universidades. Madrid.

Rodríguez Castellanos, Arturo; Araujo de la Mata, Andrés y Javier Urrutia Gutiérrez. La gestión del conocimiento científico-técnico en la universidad: Un caso y un proyecto.

OCCA (2005). Indicadores de gestión para la UNMSM. 


\section{FUENTES ELECTRÓNICAS}

www.madrimasd.org/informacionidi/ biblioteca/

Gestión del Conocimiento en Universidades y Organismos Públicos. www.invenia.es/inveniaextensions

Conocimiento Científico Técnico Universidad- Caso Proyecto.

www.ehu.es/cuadernosdegestion/documentos Gestión del Conocimiento CientíficoTécnico en la Universidad. 